\title{
Optimization of CXRS TESPEL diagnostics on LHD in the visible spectral range
}

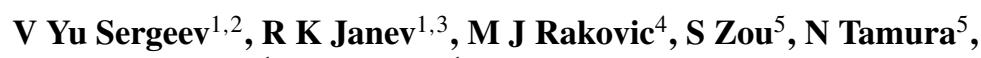 \\ K V Khlopenkov ${ }^{1}$ and S Sudo \\ ${ }^{1}$ National Institute for Fusion Science, Oroshi-Cho, 322-6, Toki, Gifu 509-5292, Japan \\ 2 State Technical University, Politechnicheskaya 29, St Petersburg, PO Box 428, 1000 Russia \\ ${ }^{3}$ Macedonian Academy of Sciences and Arts, 195251, Skopje, Macedonia \\ ${ }^{4}$ Oak Ridge National Laboratory, Oak Ridge, TN 37831-6371, USA \\ 5 The Graduate University for Advanced Studies, Hayama-Cho, Miura-gun, Kanagawa, Japan
}

Received 13 November 2001

Published 29 January 2002

Online at stacks.iop.org/PPCF/44/277

\begin{abstract}
A model for calculations of Charge eXchange Recombination (CXR) signals of impurity nuclei injected in a high-temperature plasma has been developed. The CXR signals for an Li tracer in Tracer Encapsulated Solid PELlet (TESPEL) experiments on LHD and CHS machines have been calculated. The difference of about two orders of magnitude found in the calculated signals is due to the difference both in the NBI neutral flux density and in the capture-radiation crosssections, and explains the weak $\mathrm{Li}^{3+} \mathrm{CXR}$ signals observed in the measurements on LHD.

Calculations of the CXR signals for various injected impurities have been performed for LHD conditions. The operational range of TESPEL diagnostics in the visible spectral range on LHD has been determined. For $T_{\mathrm{e}}=1-2 \mathrm{keV}$ $\left(P_{\mathrm{NBI}}=3 \mathrm{MW}\right)$ and $N_{\mathrm{e}}=(2-5) \times 10^{13} \mathrm{~cm}^{-3}$, injection of $\mathrm{F}, \mathrm{Mg}$ and $\mathrm{Al}$ as tracer materials ensures the largest CXR signals together with complete ionization times of the injected impurity which are much smaller than the impurity transport times.
\end{abstract}

\section{Introduction}

The Tracer Encapsulated Solid PELlet (TESPEL) injection method that has recently been suggested in [1] aims to provide, in a narrow region of the core plasma of a toroidal device, a controlled amount of a selected impurity for diagnostic and plasma behaviour study purposes. The method can be coupled with the Charge eXchange Recombination Spectroscopy (CXRS) diagnostic method using either a diagnostic or a heating beam. The CXRS TESPEL diagnostics is, thus, a specific version of the general CXRS method, and can be used for all plasma studies for which CXRS is usually applied (determination of ion temperature, plasma rotation, impurity transport, etc). The CXRS TESPEL method has been successfully used for $\mathrm{Li}^{3+}$ 
transport studies in TESPEL experiments with LiH tracer on CHS [2, 3] using a $36 \mathrm{keV}$ neutral $\mathrm{H}$ beam as a target for CXRS reactions where ratios of CXR signal to background in the range 2-3 were measured. Unfortunately, no detectable signals were observed in the case of LHD TESPEL experiments with $\mathrm{LiH}$ tracer where the neutral $\mathrm{H}$ beam had $150 \mathrm{keV}$ energy [3, 4].

The purpose of this paper is to explain the weak CXR $\mathrm{Li}^{3+}$ signal in LHD TESPEL experiments and to determine the operational range of the TESPEL CXRS diagnostics in the visible spectral range for the study of impurity transport in LHD using a $150 \mathrm{keV}$ neutral hydrogen atom beam.

\section{General considerations}

The basic idea of the method is illustrated in the example of the TESPEL diagnostics setup developed for LiH tracer at the Large Helical Device (LHD). LHD is a large-scale superconducting toroidal fusion facility, which is a heliotron-type device with a set of $l=2 / \mathrm{m}=10$ continuous helical coils $[5,6]$. The major and minor radii are 3.9 and $0.6 \mathrm{~m}$, respectively, and the designed toroidal magnetic field is $3 \mathrm{~T}$. LHD has a wide operational range of plasma parameters, $T_{\mathrm{e}}=1-10 \mathrm{keV}$ and $N_{\mathrm{e}}=(0.4-15) \times 10^{13} \mathrm{~cm}^{-3}$, depending on the applied heating (NBI, ECRH, ICRH) power [7]. Typical energy confinement times of the machine are about 50-300 ms [7].

In the LHD CXRS TESPEL diagnostics, a solid impurity material (tracer) is encapsulated in an outer layer of polystyrene (shell) and is accelerated in the direction of the plasma core by a pellet injector. The ablating pellet initially deposits its shell material in the plasma and then the tracer material ( $\mathrm{LiH}$ in our example) deposits itself in a narrow region of about several centimetres of the plasma core. After toroidal symmetrization and complete ionization of the tracer material, an $\mathrm{Li}^{3+}$ annular domain is created. It only slightly disturbs the plasma electron temperature and density (5-10\%), since the total amount of the particle content in both the shell and tracer material is rather small.

The radial behaviour of this localized domain can be measured by observation of CXR emission intensity $I^{Z}$ due to $\mathrm{CX}$ reactions of injected impurity nuclei (nuclear charge $Z$ ) with the neutral beam injection (NBI) hydrogen atoms (assumed to be in their ground state)

$$
\mathrm{I}^{Z+}+\mathrm{H}_{1 \mathrm{~s}} \Rightarrow \mathrm{I}^{(Z-1)+}+\mathrm{H}^{+}
$$

where $n$ and $l$ are the principal and orbital angular quantum numbers of captured electron on the ion. For ions with charge $Z$, the electron capture takes place preferentially on excited energy levels with $n \approx Z$. For $Z \geqslant 2$, these are the excited product ion levels that radiate and the emission of which gives the basis for the CXRS diagnostics of plasma impurities (as well as other plasma parameters).

The CXR signal on LHD is measured within a certain visible spectral range. Therefore, the perturbation of the signal caused by the visible continuum radiation is possible mainly due to ablatant created by the shell material. The amount of tracer material is about two orders of magnitude less than the amount of shell particles. Estimates show that the input of tracer material in the perturbation of continuum radiation can be neglected up to the tracer charge values of $Z \approx 17-18$. Besides, a background (reference) emission can appear due to the charge exchange between fully ionized ions and thermal neutrals that come from the plasma edge where the ion temperature is low [8]. For these reasons, the CXRS system developed for measurements of plasma poloidal rotation on LHD [8] is used. In this system, the CXRS detector array in the NBI port is arranged together with the reference detector array that has a geometry identical to the CXRS detectors in the port without NBI. Signals from the detectors via optical fibres are transferred to the entrance of a set of photomultipliers. These 
photomultipliers are equipped with sets of interference filters that provide measurements of a narrow specific spectral range $(\sim 1 \mathrm{~nm})$ containing the line intensity of H-like ions of the injected impurity. Thus, subtraction of the reference signals from the CXR detector signals results in pure CXR signals due to reaction (1). By measuring the time interval $\Delta t$ between the maxima of CXR signals with a radial $\Delta r$ displacement, one can derive the effective diffusion coefficient of tracer impurity, averaged over the radial range $\Delta r$.

The key parameter of any CXRS diagnostics is the cross-section of reaction (1). In figure 1 the reduced total $\mathrm{CX}$ cross-section $\sigma^{\mathrm{CXtot}} / Z$ versus the reduced NBI energy $E / Z^{3 / 7}$, taken from [9], is shown. The arrows in the figure indicate the conditions for different energy $E$ of $\mathrm{NBI}$ and $Z$ of tracer material corresponding to the CHS $(E=36 \mathrm{keV})$ and LHD $(E=150 \mathrm{keV})$ TESPEL experiments. It can be seen that the CX total cross-section drastically depends on $E$ and $Z$. For an Li tracer, the $\sigma^{\text {CXtot }}$ value for CHS is 30 times larger than that for LHD NBI energy. As is obvious from figure 1, at the fixed NBI energy in LHD (150 keV), one should have to select higher $Z$ tracer materials (see points for $\mathrm{F}, \mathrm{Si}$, Ti shown in figure 1) in order to reach the reduced energy region of high reduced $\mathrm{CX}$ cross-sections.

However, the increase of the atomic number of the tracer material leads to additional requirements. Namely, the ionization of tracer atoms can be incomplete at a given electron temperature $T_{\mathrm{e}}$. Hence, the density $N_{Z}$ of fully stripped impurity ions can differ from the density $N_{\text {imp }}$ of the injected impurity by a factor equal to the ionization degree $\alpha_{Z}=N_{Z} / N_{\text {imp }}$. The CXR signal decreases with a decrease of the density of fully stripped ions, which may limit the CXR observations. Another limitation arises from the requirement that the time $\tau_{\text {ion }}$ for complete ionization of injected impurity has to be significantly smaller than the characteristic diffusion time $\tau_{\text {diff }}$. For a light material like Li, the relationships

$$
\alpha_{Z} \approx 1, \quad \tau_{\text {ion }} \ll \tau_{\text {diff }}
$$

are easily fulfilled. For higher- $Z$ tracers, however, these conditions may be violated and the ionization balance processes of these impurity ions have to be fully taken into consideration.

The apparatus for the TESPEL diagnostics on LHD has been developed for the visible spectral range. It is well known that in high- $Z$ ions only transitions with a change of principal quantum number $n$ by $1(\Delta n=1)$ can provide wavelengths in the visible domain. For the radiation in the visible spectral range, the principal quantum number $n_{\mathrm{v}}$ of radiating level and the ionic charge $\mathrm{Z}$ are related to each other by the following simple relation:

$$
n_{\mathrm{v}} \approx 2.2 \times Z^{2 / 3} \text {. }
$$

We have obtained relation (3) by taking into account the fact that the visible radiation should be as close as possible to its lower margin $(\lambda \sim 400 \mathrm{~nm})$, since the radiating level $n_{\mathrm{v}}$ is then

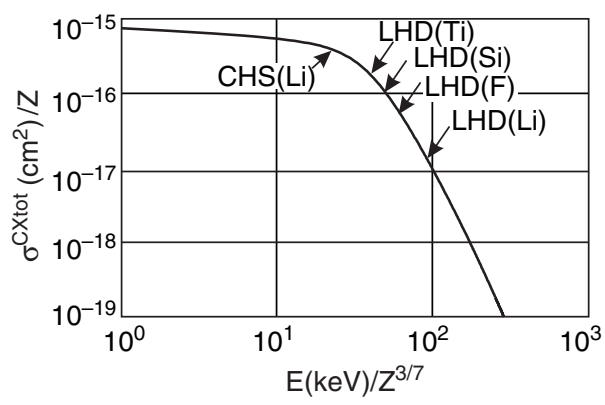

Figure 1. Reduced total $\sigma^{\mathrm{CXtot}} / Z \mathrm{CX}$ cross-section of impurity nuclei with charge $Z$ versus reduced NBI energy $E / Z^{3 / 7}$. 
closer to the maximum of $n$-distribution of captured electrons in reaction (1) for collision (or beam) energies above $100 \mathrm{keV}$ (see below). Relation (3) indicates that when using high- $Z$ tracer elements, one has to use the radiation from the high- $n$ levels while remaining within the visible spectral range. For instance, the radiation due to electron transition from $n_{\mathrm{v}}=17$ to $n_{\mathrm{v}}-1=16$ lies in the visible spectral range if $Z=22$ (i.e. the tracer has to be titanium). For collision energies above $100 \mathrm{keV}$, the total electron capture cross-section is distributed among many quantum levels of the product ion, resulting in relatively small partial $n$-CX crosssections, especially for the higher $n$-values of the distribution. The $n$-distribution of captured electrons at these collision energies has a maximum at approximately $Z^{0.815}$ (see below). Therefore, although the total CX cross-section increases with $Z$, it does not automatically guarantee large $n$-partial cross-section for $n=n_{\mathrm{v}}$. Moreover, the radiation takes place due to transitions between given initial and final $n, l$-substates, which means that the partial $n$-CX cross-section has to be distributed among all the $n, l$-substates within a given $n$-energy level, which further reduces the portion of the total cross-section that contributes to the CXR signal. Therefore, one should consider the population of the specific sublevels $n, l$ very carefully, with inclusion of not only the CX process (1) but also of all other processes contributing to the population and depopulation of radiating $n, l$-sublevel.

\section{Model}

The consideration of a wide range of nuclear charges (varieties of injected impurities) with the purpose of optimization of the CXR signal (with respect to the optimal choice of tracer impurity for the given range of LHD plasma parameters and given beam energy) requires a simplification of the collisional-radiative (CR) set of equations for calculation of the CXR signal, as well as the corona equilibrium (CE) set of equations for the ionization balance, in order to evaluate conditions (2).

\subsection{Calculation of CXRS signal}

An excited state of an $\mathrm{H}$-like ion in high-temperature plasmas can be populated by radiative recombination with a plasma electron, by charge exchange of a parent nucleus on the beam atoms, by radiative decay and collisional de-excitation of higher states and by collisional excitation of lower states. It can be depopulated by radiative decay and collisional de-excitation to lower states and by collisional ionization.

To calculate the CXR signal, the set of CR equations was simplified by taking into account the following dominant processes that populate the ' $n l$ '-states of hydrogen-like impurity ions with density $N_{n l}^{Z-1}$ :

(a) CX reaction (1) with partial cross-section $\sigma_{n l}^{C X}$ for populating a specific ' $n l$ '-state;

(b) radiative decay cascade from all levels with $n^{\prime}>n$ to the level $n$;

(c) radiative decay transitions from the level $n$ to all levels with $n^{\prime}<n$.

The applicability of this approach is discussed in the Appendix. It is shown that for large $Z$ and $n$ of interest here, the processes of radiative recombination of nuclei, excitation, de-excitation and ionization of $\mathrm{H}$-like ions of injected impurity are negligible in comparison with the processes listed above. Thus, the set of equations for population of H-like ion density $N_{n l}^{Z-1}$ in the specific state $n l$ is

$$
\frac{\mathrm{d} N_{n l}^{Z-1}}{\mathrm{~d} t}=N^{Z} N_{\mathrm{H}} v_{\mathrm{H}} \sigma_{n l}^{\mathrm{CX}}+\sum_{n^{\prime}>n, l^{\prime}} N_{n^{\prime} l^{\prime}}^{Z-1} A_{n^{\prime} l^{\prime}}^{Z-1}-N_{n l}^{Z-1} \sum_{n^{\prime}<n, l^{\prime}} A_{n l, n^{\prime} l^{\prime}}^{Z-1} .
$$


Here, $N^{Z}$ is the density of impurity nuclei, $N_{\mathrm{H}} v_{\mathrm{H}}$ is the flux of NBI atoms, $l^{\prime}=l \pm 1$ (that follows from the selection rule for dipole-allowed transitions) and $A_{n l, n^{\prime} l^{\prime}}^{Z-1}$ is the transition probability for spontaneous $n l \rightarrow n^{\prime} l^{\prime}$ radiative decay in the H-like ions.

The photon volumetric source of CX reaction in the visible spectral range is due to the transitions $n l, \rightarrow(n-1) l^{\prime}$ only,

$$
\frac{\mathrm{d} I_{n l(n-1) l^{\prime}}^{Z-1}}{\mathrm{~d} V}=\frac{1}{4 \pi} N_{n l}^{Z-1}\left(A_{n l,(n-1)(l+1)}^{Z-1}+A_{n l,(n-1)(l-1)}^{Z-1}\right) \quad\left(\text { photons }^{-3} \mathrm{~s}^{-1} \mathrm{strd}^{-1}\right)
$$

The solution of equations (4) was obtained by using the approach described in [10]. Thus, for the steady-state condition $t \gg 1 / \min \left(A_{n l, n^{\prime} l^{\prime}}^{Z-1}\right)$, from the set of equations (4) one can obtain

$$
N_{n l}^{Z-1}=\frac{N^{Z} N_{\mathrm{H}} v_{\mathrm{H}}\left(\sigma_{n l}^{\mathrm{CX}}+\sum_{n^{\prime}>n} C_{n^{\prime}, n} \sigma_{n^{\prime}, l}^{\mathrm{CX}}\right)}{\sum_{n^{\prime}<n} A_{n l, n^{\prime} l^{\prime}}^{Z-1}},
$$

where $C_{n^{\prime}, n}$ is the cascade matrix from all levels $n^{\prime}>n$ calculated in [10]. We have assumed that the original $l$-distribution due to $C X$ reaction is not significantly disturbed during cascade processes. Substituting equation (6) into equation (5), and making a summation over the angular quantum numbers $l$, we obtain

$$
\frac{\mathrm{d} I_{n,(n-1)}^{Z-1}}{\mathrm{~d} V}=\frac{1}{4 \pi} N^{Z} N_{\mathrm{H}} v_{\mathrm{H}}\left\langle\sigma_{n l}^{\mathrm{CX}}\right\rangle \quad\left(\text { photons } \mathrm{m}^{-3} \mathrm{~s}^{-1} \mathrm{strd}^{-1}\right)
$$

where $\left\langle\sigma_{n,(n-1)}^{\mathrm{CX}}\right\rangle$ is the capture-radiation cross-section for the transition $n \rightarrow n-1$,

$$
\left\langle\sigma_{n l}^{\mathrm{CX}}\right\rangle=\sum_{l=0}^{n-1}\left(\sigma_{n l}^{\mathrm{CX}}+\sum_{n^{\prime}>n} C_{n^{\prime}, n} \sigma_{n^{\prime}, l}^{\mathrm{CX}}\right) \times P_{n l,(n-1) l^{\prime}}
$$

and $P_{n l,(n-1) l^{\prime}}$ is the radiation probability, i.e. the ratio of $A_{n l, n^{\prime} l^{\prime}}^{Z-1}$ decay probabilities for the transitions $n l \Rightarrow(n-1) l^{\prime}\left(l^{\prime}=l \pm 1\right)$ to the total probability of radiative decay of the level $n$ :

$$
P_{n l,(n-1) l^{\prime}}=\frac{A_{n l,(n-1)(l+1)}^{Z-1}+A_{n l,(n-1)(l-1)}^{Z-1}}{\sum_{n^{\prime}<n} A_{n l, n^{\prime} l^{\prime}}^{Z-1}} .
$$

To calculate the radiation photon flux measured in experiments by a detector with a detection area $S_{\mathrm{d}}$ and at a distance $R_{\mathrm{d}}$ from the source, the volumetric source of CX photon flux emission (7) has to be integrated over the whole observation volume $V_{\text {obs }}$ :

$$
I_{n,(n-1)}^{Z-1}=\int_{V_{\mathrm{obs}}} \frac{S_{\mathrm{d}}}{4 \pi R_{\mathrm{d}}^{2}} N^{Z} N_{\mathrm{H}} v_{\mathrm{H}}\left\langle\sigma_{n,(n-1)}^{\mathrm{CX}}\right\rangle \mathrm{d} v \quad \text { (photons s }{ }^{-1} \text { ). }
$$

One can see from equation (10) that for a fixed NBI energy and power, and for a given geometry of TESPEL experiment, the CXR signal is proportional to the $\left\langle\sigma_{n, n-1}^{\mathrm{CX}}\right\rangle$ captureradiation cross-section multiplied by the density $N^{Z}$ of the nuclei. From a methodological point of view, we should keep the amount of injected impurity at a fairly low level. Therefore, key parameters are the dependencies of $\sigma_{n l}^{\mathrm{CX}}$ partial cross-section and $P_{n l,(n-1) l^{\prime}}$ radiation probability for $\mathrm{H}$-like atomic systems on the $n, l$ quantum numbers (see equations (8) and (9)).

\subsection{Partial CX cross-sections and radiation probability}

The $n$ - and $n, l$-partial CX cross-sections for $150 \mathrm{keV}$ NBI energy in LHD were calculated for the fully stripped ions with $Z=3,6,9,12,14,18,22$ by using the classical trajectory 
Monte Carlo (CTMC) method [11]. Since the calculations for any $Z$ are highly timeconsuming, the following scaling fit-functions for $\sigma_{n l}^{\mathrm{CX}}$ and $\sigma_{n}^{\mathrm{CX}}=\sum_{l=0}^{n-1} \sigma_{n l}^{\mathrm{CX}}$ were determined as (on the basis of CTMC data for $E=150 \mathrm{keV}$ )

$$
\begin{aligned}
& \sigma_{n}^{\mathrm{CX}}(n, Z)=1.1 \times 10^{-17} \times Z^{3 / 2} \frac{\exp \left(-1.35 /\left(n / Z^{0.815}\right)^{2.3}\right)}{\left(n / Z^{0.815}\right)^{3}}\left(\mathrm{~cm}^{2}\right) \\
& \sigma_{n_{\mathrm{v}}, l}^{\mathrm{CX}}(l, Z)= \sigma_{n}^{\mathrm{CX}}\left(n_{\mathrm{v}}, Z\right) \frac{9.5}{Z^{0.75}}\left(\left(\frac{l}{Z}\right)^{4}+\frac{l}{8 Z}\right) \\
& \times \exp \left(-\left(\frac{1.43}{Z}\left(1+3 \times 10^{-6} \times Z^{3}\right)\right)^{8+Z^{2.5} / 200}\right) .
\end{aligned}
$$

The results of CTMC calculations and those produced by the scaling functions (11) and (12) for some $Z$-ions from the $Z=3-22$ range are shown in figure 2 . For the $Z=6-22$ range, the principal quantum numbers $n_{\mathrm{v}}$ for the visible domain are shown by the hatched area in figure 2(a), together with a vertical segment for $n_{\mathrm{v}}$ corresponding to the Li tracer material.

(a)

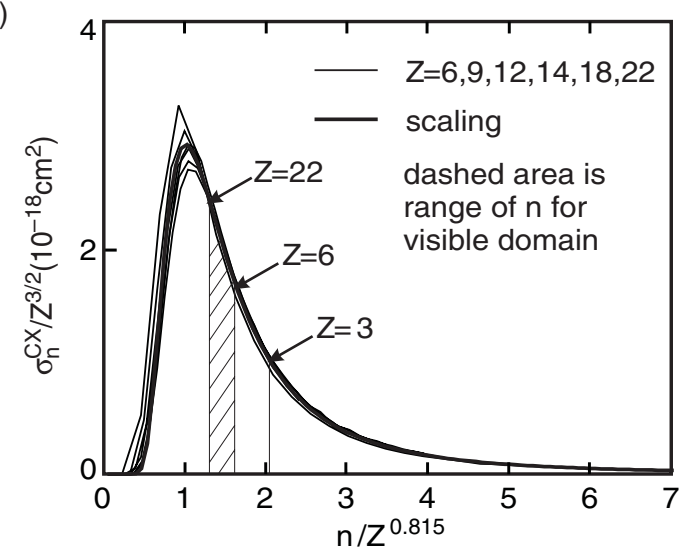

(b)

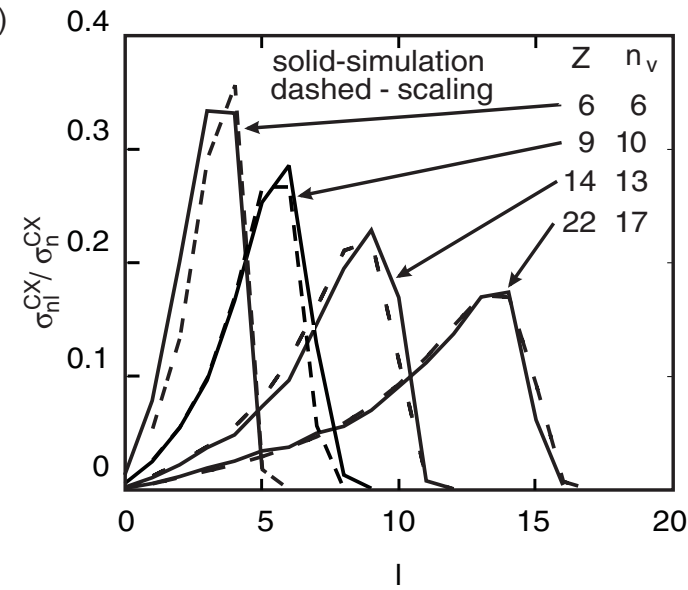

Figure 2. Dependencies of partial total $\sigma_{n}^{\mathrm{CX}}(a)$ and $\sigma_{n_{\mathrm{v}} l}^{\mathrm{CX}}(b)$ versus the quantum numbers $n, l$, respectively. 
One can see that the partial cross-sections $\sigma_{n}^{\mathrm{CX}}$ corresponding to the visible domain increase with increasing $Z$ and their values are not very far from the maximum of $n$-distribution.

It is seen from figure $2(b)$ that with increasing $Z$ the $\sigma_{n l}^{C X}$ dependencies have a tendency to be closer to the statistical distribution $\sigma_{n l}^{\mathrm{CX}} / \sigma_{n}^{\mathrm{CX}}=(2 l+1) / n^{2}$ over $l$. It seems that the $n, l$-distribution of captured electrons is closer to a situation when the 'strong mixing' of Stark states due to the rotation of the internuclear axis [11] takes place as the population mechanism of different $n, l$-substates in reaction (1). However, for light- $Z$ materials the maximum of the $l$-distribution of captured electrons on a given level $n$ shifts towards the lower $l$-values (the case of 'weak mixing' [11]), and this situation is unfavourable for the magnitude of CXR signal if one takes into account that the radiation probability $P_{n l,(n-1) l^{\prime}}$ (a multiplier in equation (8) for the capture-radiation cross-section) has an opposite tendency with increasing $l$, as shown below.

The radiation probability for $\mathrm{H}$-like atomic systems was calculated for various $Z$-ions and $n, l$ quantum numbers according to the prescription given in [12]. The calculated $P_{n l,(n-1) l^{\prime}}$ dependencies on the angular quantum number difference $l-(n-1)$, for the given $n$, are shown in figure 3. The values of $n_{\mathrm{v}}$ for different $Z$ in figure 3 correspond to the transitions in the visible domain (equation (3)).

\subsection{Ionization balance of high- $Z$ ions}

The ionization degrees $\alpha_{Z}$ have been calculated for a wide range of $Z$-impurities using the simplified CE set of equations (13) given below. The number of impurity nuclei has to be very large (preferably close to the amount of injected atoms) for a CXR measurement. It is well known that the ionization degree becomes large for temperatures $T_{\mathrm{e}} \geqslant I_{\text {ion }}$, where $I_{\text {ion }}$ is the ionization potential of H-like ions. Therefore, one can take that the densities of all states, beginning from the Li-like one, are zero, which is quite an acceptable approximation for the temperature range of interest here. This gives the well-known CE result [10]

$$
\frac{N^{Z}}{N^{Z-1}}=\frac{S^{Z-1}}{R^{Z}}, \quad N^{q} \equiv 0, \quad q=Z-3, Z-4, \ldots, 0,
$$

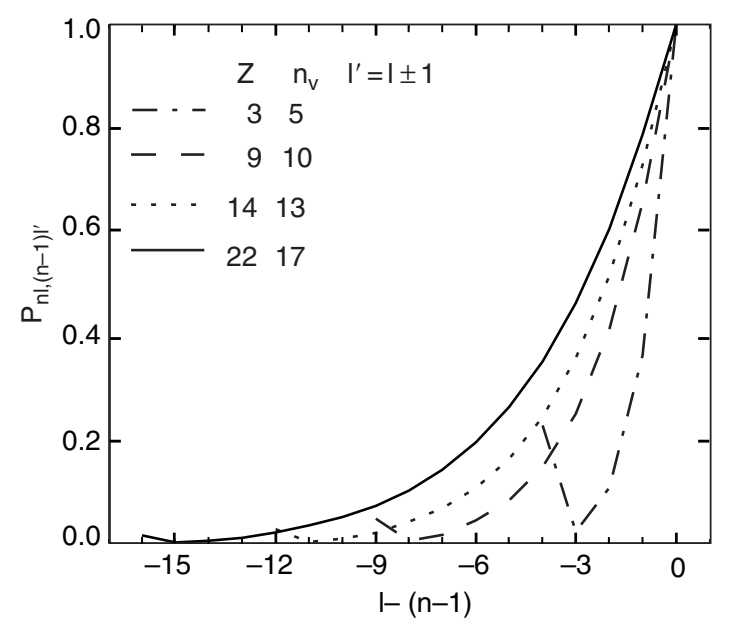

Figure 3. Radiation probability $P_{n l,(n-1) l^{\prime}}$ from the $n l$-substate to the $(n-1) l^{\prime}$ substates versus the difference of angular momentum quantum numbers: $l-(n-1)$. 
where $S^{Z-1}$ and $R^{Z}$ are the effective ionization and recombination rate coefficients of the indicated ions. Thus, only the nuclei, $\mathrm{H}$ - and He-like ions were taken into account in our model. The ionization rate coefficients presented in the Appendix were calculated for the ground states $(n=1)$ only, since due to the large radiative decay probabilities a small population of higher principal quantum numbers is expected. The results of calculations of effective ionization rate coefficient of $\mathrm{H}$-like ions, performed within the CR model [10], confirm the validity of this approach. The rate coefficients for other processes, needed in the ionization balance calculations and used in this paper, are given in the Appendix. Because of small values of the ratio $N_{\mathrm{H}} / N_{\mathrm{e}} \approx(1-3) \times 10^{-6}$, we have neglected the $\mathrm{CX}$ recombination term in the ionization balance equations. In that case, the calculation of $\alpha_{Z}$ becomes independent of the electron density.

To validate our simplified calculations of $\alpha_{Z}$, we compare the obtained results with those obtained using the MIST impurity transport code [13], in which all ionization states are taken into account in equations (13). The comparison is shown in figure 4. It is seen that the simplified model slightly underestimates the $\alpha_{Z}$ values, and the difference increases with increasing $Z$ at fixed $T_{\mathrm{e}}$. However, these discrepancies are not larger than $10-15 \%$ even for high- $Z$ material like Ti.

In order to estimate the time needed for complete ionization $\tau_{\text {ion }}$, we have used the fact that the ionization potentials of $\mathrm{H}$ - and He-like ions of injected impurities are close to each other and these values are much larger than the ionization potentials of lower ionization states. Thus, $\tau_{\text {ion }}$ can be estimated as the sum of ionization times of $\mathrm{H}$ - and He-like ions for the temperature range of interest, $T_{\mathrm{e}} \geqslant I_{\text {ion }}$. Using the MIST code, we have calculated the time $\tau_{\text {equil }}$ needed to reach the ionization equilibrium of an impurity with charge $Z$ at the given electron temperature $T_{\mathrm{e}}$. The results of these calculations are shown in figure 5 together with the $\alpha_{Z}$ values from figure 4. It can be seen that for temperatures $T_{\mathrm{e}} \geqslant I_{\text {ion }}$, the times $\tau_{\text {ion }}$ calculated by our CE model are close to $\tau_{\text {equil }}$ obtained from the MIST code calculations. The decrease of $\tau_{\text {equil }}$ for temperatures lower than the corresponding ionization potential can be explained by the fact that for these conditions the equilibrium is determined by the lower ionization states, with correspondingly lower ionization times.

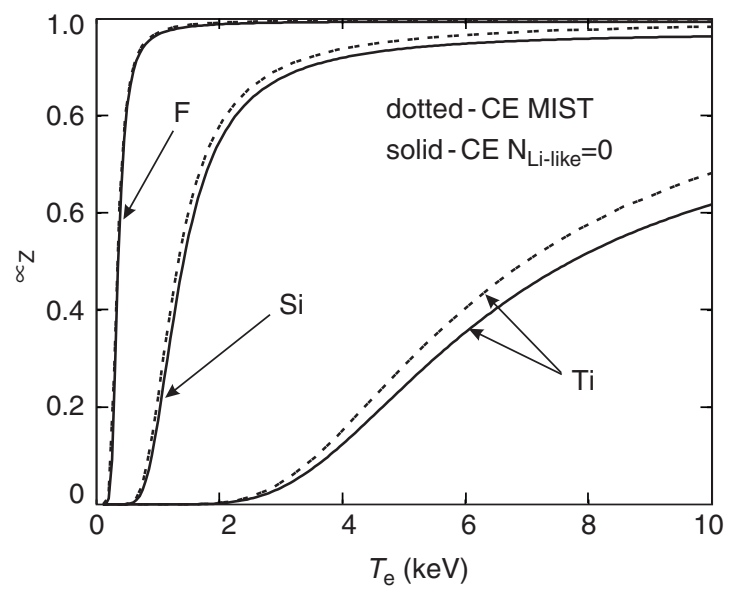

Figure 4. Complete ionization degree $\alpha_{Z}$ versus electron temperature calculated by the simplified model (solid curves) and by the MIST code (dashed curves) for F, Si, Ti. 


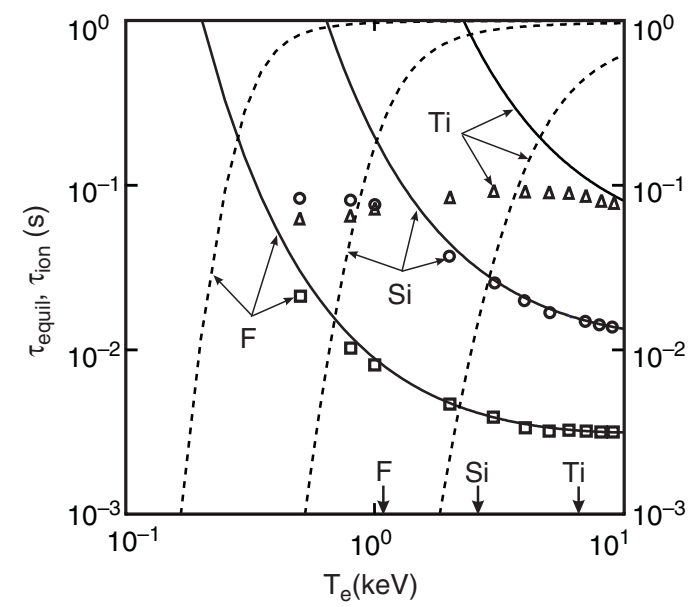

Figure 5. Complete ionization time $\tau_{\text {ion }}$ calculated by the simplified model (solid curves) and equilibrium time $\tau_{\text {equil }}$ calculated by the MIST code (triangles for $\mathrm{Ti}$, circles for $\mathrm{Si}$, squares for F) versus electron temperature at $N_{\mathrm{e}}=10^{13} \mathrm{~cm}^{-3}$. Dotted curves are $\alpha_{Z}$ dependencies on $T_{\mathrm{e}}$ from figure 4 . Arrows to the $x$-axis show the ionization potentials of $\mathrm{H}$-like ions of indicated impurities.

\section{Results of calculations and discussion}

\subsection{Calculations of CXRS signal for CHS and LHD}

In figure 6, the partial $\mathrm{CX}$ radiation cross-sections for $\mathrm{Li}$ material as a tracer for $\mathrm{CHS}$ $\left(E_{\mathrm{NBI}}=36 \mathrm{keV}\right)$ and LHD $\left(E_{\mathrm{NBI}}=150 \mathrm{keV}\right)$ cases are shown, together with the $l$-dependence of radiation probability for $\mathrm{H}$-like $\mathrm{Li}$ ion. The reasons resulting in the large difference of $\mathrm{Li}$ capture-radiation cross-sections in these two experiments are evident from figure 6 . There are small absolute values of CX cross-section and unfavourable dependence of the partial crosssection on the angular quantum number $l$ relative to $l$-dependence of the radiation probability mentioned above. Results of evaluations of CXR signals of $\mathrm{Li}^{3+}$ ions for TESPEL experimental conditions on these two machines are presented in table 1 . Here, $R_{\mathrm{p}}$ is the pellet radius and $S_{\mathrm{NBI}}$ is NBI cross-section area.

The most different input parameters of CXR signal evaluations by means of equation (10) in the two machines are underlined in the table. It is seen that small values of both captureradiation cross-section and NBI density flux result in two order of magnitude smaller CXR signal $I_{5,4}^{2}$ values for the LHD case with respect to CHS. This explains why no detectable difference between the CXRS and reference detector signals has been observed in LHD TESPEL experiments with LiH tracer.

\subsection{Calculations of CXRS signal for various $Z$ tracer materials in $L H D$}

The model developed in section 3 can be used for calculations of CXRS signal for various $Z$ tracer materials in the LHD case with a $150 \mathrm{keV}$ NBI energy. The capture-radiation crosssection for the visible spectral range, normalized to its $4.7 \times 10^{-19} \mathrm{~cm}^{2}$ value for $\mathrm{Li}$, is shown in figure 7 versus the tracer nuclear charge $Z$. The analysis of figure 7 confirms the preliminary conclusion made previously on the basis of considerations of total cross-section dependence on $Z$ (that one should use a higher- $Z$ material for TESPEL injection into LHD plasmas). The 


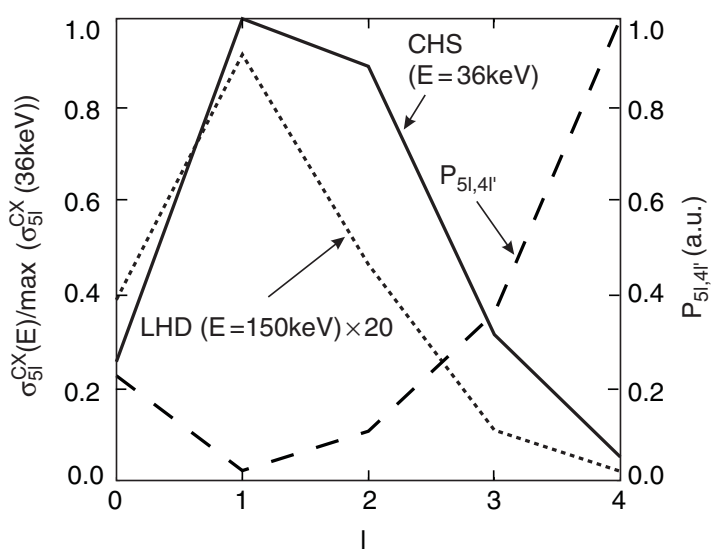

Figure 6. Partial CX radiation cross-sections $\sigma_{5 l}^{\mathrm{CX}}(E)$ for Li versus $l$ for CHS (solid curve) and for LHD multiplied by a factor of 20 (dotted curve). The dashed curve is the $l$-dependence of radiation probability for $\mathrm{H}$-like $\mathrm{Li}$ ions. The cross-sections $\sigma_{5 l}^{\mathrm{CX}}(E)$ are normalized to the maximal $\sigma_{5 l}^{\mathrm{CX}}$ $(36 \mathrm{keV})$ value.

Table 1. Evaluations of $i^{3}$ CXR signals for CHS and LHD

\begin{tabular}{lcc}
\hline Parameter & CHS & LHD \\
\hline$P_{\mathrm{NBI}}(\mathrm{MW})$ & 0.7 & 3 \\
$E_{\mathrm{NBI}}(\mathrm{keV})$ & 36 & 150 \\
$S_{\mathrm{NBI}}\left(\mathrm{m}^{2}\right)$ & 0.016 & 0.36 \\
$J / e=n_{\mathrm{H}} v_{\mathrm{H}}\left(10^{17} \mathrm{~cm}^{-2} \mathrm{~s}^{-1}\right)$ & $\underline{5}$ & $\underline{0.2}$ \\
$\left\langle\sigma_{5,4}^{\mathrm{CX}}\right\rangle\left(10^{-17} \mathrm{~cm}^{2}\right)$ & $\underline{1.47}$ & $\underline{0.047}$ \\
$R_{\mathrm{p}}(\mathrm{mm})$ & 0.025 & 0.1 \\
$N_{Z}=N_{\mathrm{Li}}\left(10^{9} \mathrm{~cm}^{-3}\right)$ & 5 & 10 \\
$V_{\mathrm{obs}}\left(\mathrm{cm}^{3}\right)$ & $\underline{10}$ & $\underline{60}$ \\
$\omega_{\mathrm{det}}=\left(S_{\mathrm{d}} / R_{\mathrm{d}}\right)^{2}\left(10^{-4}\right)$ & 1.5 & 1.0 \\
$I_{5,4}^{2}\left(10^{5}\right.$ photons s$\left.{ }^{-1}\right)$ & 44 & 0.44 \\
\hline
\end{tabular}

evaluated capture-radiation cross-sections for $Z \geqslant 10$ are 30-100 times larger than that for Li tracer material.

As was mentioned above and can be seen from equation (10), at a fixed geometry of the experiment, NBI energy and power, the CXR signal is proportional to both $\left\langle\sigma_{n,(n-1)}^{\mathrm{CX}}\right\rangle$ and the density of injected impurity nuclei, $N^{Z}$. The last quantity can differ from the density $N_{\text {imp }}$ of injected impurity atoms by (ionization degree) $\alpha_{Z}$ times. Hence, the CXR signal increases with an increase of the product parameter $\Theta=\left\langle\sigma_{n,(n-1)}^{\mathrm{CX}}\right\rangle \times \alpha_{Z}$.

The functional dependence of this parameter on $Z$ was calculated using the simplified ionization balance model and is shown in figure $8(a)$ for three different electron temperatures. The dependence of parameter $\Theta$ on $Z$ has a maximum because $\left\langle\sigma_{n,(n-1)}^{\mathrm{CX}}\right\rangle$ increases and $\alpha_{Z}$ decreases with increasing $Z$, and it depends on electron temperature. These $Z$-dependencies can be reduced (approximately) to one curve by dividing the product $\Theta$ by $T_{\mathrm{e}}^{3 / 4}$ and its argument $Z$ by $T_{\mathrm{e}}^{1 / 3}$. The reduced $\Theta$ from figure $8(a)$ versus reduced tracer charge are shown in figure $8(b)$ together with their fit $f_{\mathrm{CXR}}(x)$ :

$$
f_{\mathrm{CXR}}(x)=\frac{8.5 x^{2.2}}{x^{18}+100}, \quad x=\frac{Z}{T_{\mathrm{e}}^{1 / 3}(\mathrm{eV})} .
$$




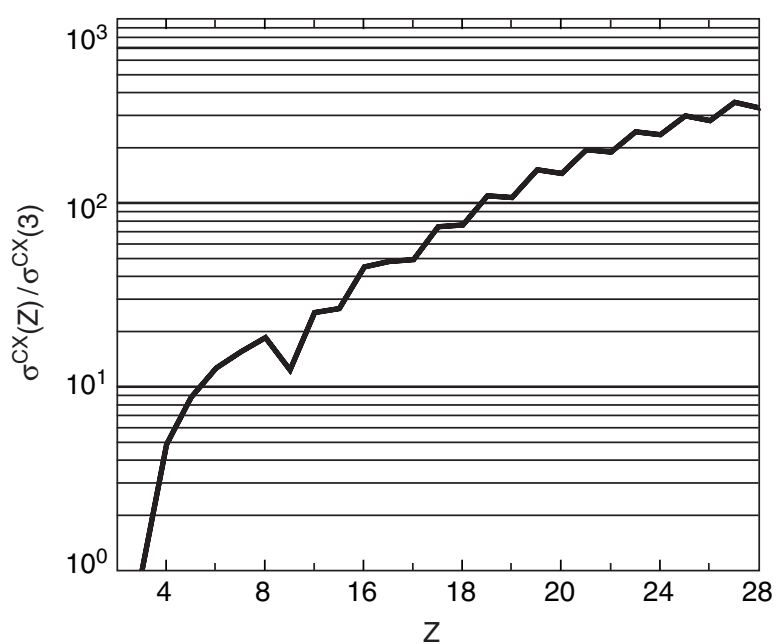

Figure 7. Capture-radiation cross-sections for the visible spectral range, normalized at its value for $\mathrm{Li}$, versus $Z$ for the LHD case with a $150 \mathrm{keV} \mathrm{NBI}$ energy.

(a)

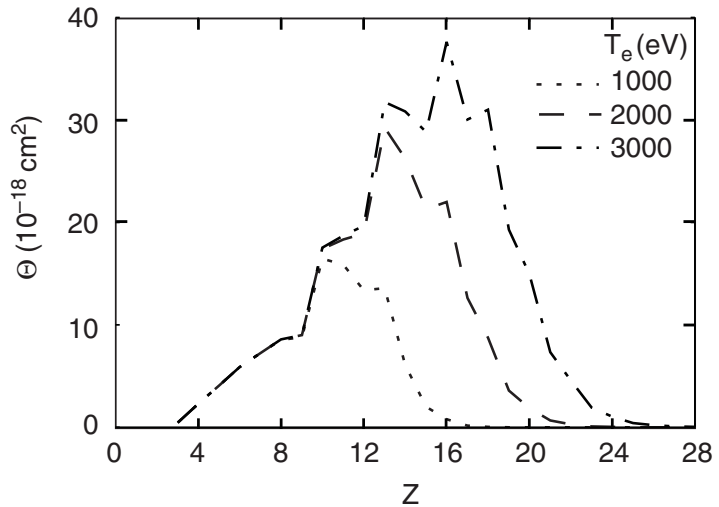

(b)

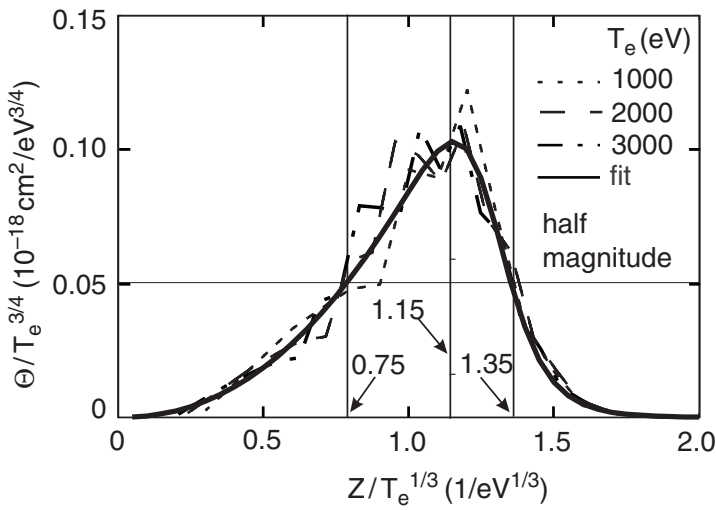

Figure 8. Product CXR parameter $\Theta$ versus $Z$ for different electron temperatures $(a)$ and reduced product CXR parameters versus reduced $Z(b)$. 
The observed scaling property of the product $\Theta$ leads to a simple relationship between the electron temperature and the charge $Z_{\text {opt }}$ for which optimal observation conditions of the maximum CXR signal can be achieved. In a similar way, one can introduce a range of tracer materials with $Z$ ranging from $Z_{\min }$ to $Z_{\max }$ by the requirement that the CXR signal level should not be smaller than half its maximum amplitude (see figure $8(b)$ ). These conditions are expressed as

$Z_{\mathrm{opt}}=1.15 T_{\mathrm{e}}^{1 / 3}(\mathrm{eV}), \quad Z_{\min }=0.75 T_{\mathrm{e}}^{1 / 3}(\mathrm{eV}), \quad Z_{\max }=1.35 T_{\mathrm{e}}^{1 / 3}(\mathrm{eV})$.

\subsection{Operational range of TESPEL diagnostics in $L H D$}

Let us specify condition (2) by requiring that the complete ionization time $\tau_{\text {ion }}$ has to be three times smaller than the characteristic diffusion time $\tau_{\text {diff }}$. For a typical impurity diffusion coefficient $D \approx 2 \times 10^{3} \mathrm{~cm}^{2} \mathrm{~s}^{-1}$, measured in CHS and LHD (see [2,3]), and a typical distance $\Delta r \geqslant 12 \mathrm{~cm}$ between CXRS detector channels on LHD and the tracer deposition radius, $\tau_{\text {diff }}$ can be estimated as $\tau_{\text {diff }}=\Delta r^{2} / 4 D=12^{2} / 4 \times 2 \times 10^{3} \approx 18 \mathrm{~ms}$. Thus, we have

$$
\tau_{\text {ion }}\left(Z, T_{\mathrm{e}}, N_{\mathrm{e}}\right) \leqslant \frac{1}{3} \tau_{\text {diff }} \approx 6 \mathrm{~ms} .
$$

Finally, condition (15) for the CXR signal level and condition (16) for complete ionization of injected impurity lead to the establishment of a certain operational range of TESPEL diagnostics for studies of the fully stripped impurity transport in LHD at the NBI energy of $150 \mathrm{keV}$. These limits are shown in figure 9. The black thick line and error bars represent equations (15) for $Z_{\mathrm{opt}}$ and $Z_{\min }, Z_{\max }$, respectively. Conditions (16) are shown by four curves for different electron plasma densities in the $10^{13}-10^{14} \mathrm{~cm}^{-3}$ range. The limits shown in figure 9 can be easily used for choosing the TESPEL tracer material. The choice should be made for materials with atomic number within the error bars, preferably closer to the black thick curve corresponding to the maximum CXR signal, and below the curve which represents the condition on ionization time for a given electron plasma density. For example, for $T_{\mathrm{e}}=1-2 \mathrm{keV}\left(P_{\mathrm{NBI}}=3 \mathrm{MW}\right)$ and $N_{\mathrm{e}}=(2-5) \times 10^{13} \mathrm{~cm}^{-3}$, injection of $\mathrm{F}, \mathrm{Mg}$ and $\mathrm{Al}$ as a tracer material can be considered as optimal from the point of view of high CXR signals. For these conditions, the ratio of CXR signal to the background (perturbed continuum radiation)

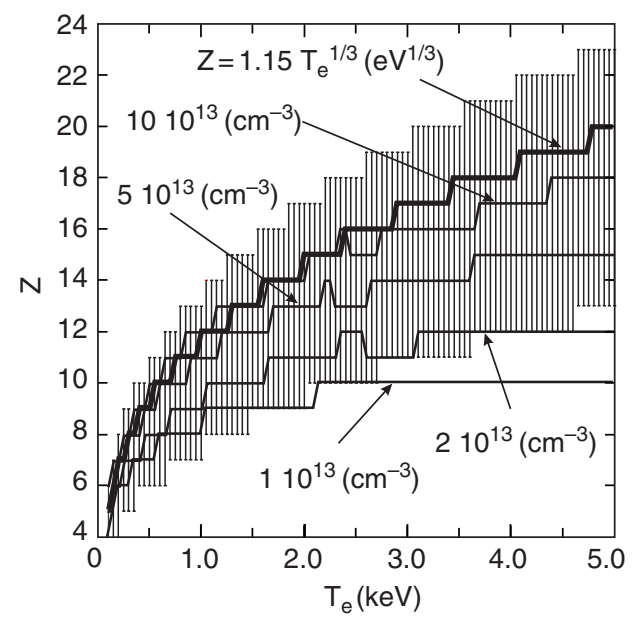

Figure 9. Operational range of TESPEL diagnostics in the visible spectral range for studies of impurity transport in the LHD machine with an NBI energy of $150 \mathrm{keV}$. 
is expected to be at the same detectable range as was in CHS TESPEL experiments with Li tracer.

\section{Conclusions}

A model for calculations of CXR signals of impurities injected in high-temperature plasmas has been developed. For that purpose, a simplified collisional-radiative model for calculations of the CXR signal and the corona equilibrium set of equations for ionization balance were solved taking into account the dominant physical processes involved. Scaling relationships for the partial CX cross-sections with respect to both the principal and angular quantum numbers (as the main input parameter in the model) have been established on the basis of numerical results of CTMC CX cross-section calculations for the $\mathrm{H}$ beam energy of $150 \mathrm{keV}$.

The CXR signals for Li tracer in TESPEL experiments on LHD and CHS machines have been calculated. The difference of about two orders of magnitude in the calculated signals due to differences in both the NBI neutral flux density and capture-radiation cross-section explains the weak $\mathrm{Li}^{3+}$ CXR signals in LHD.

The calculations of CXR signals for various injected impurities in a high-temperature plasma have been performed for the LHD conditions. Conditions have been established which give the maximum CXR signal and a complete ionization time that is much smaller than the impurity transport time. These conditions determine the operational range of the CXRS TESPEL diagnostics in the visible spectral range on LHD for the $150 \mathrm{keV}$ beam energy. At $T_{\mathrm{e}}=1-2 \mathrm{keV}\left(P_{\mathrm{NBI}}=3 \mathrm{MW}\right)$ and $N_{\mathrm{e}}=(2-5) \times 10^{13} \mathrm{~cm}^{-3}$, injection of $\mathrm{F}, \mathrm{Mg}$ or $\mathrm{Al}$ as tracer materials for LHD is suggested as optimal.

The approach developed in this paper can be applied for optimization of CXR measurements of injected impurities also for other NBI energies and/or for the VUV region of observation.

\section{Acknowledgments}

The authors thank Dr H Funaba for the help with running the MIST code and Dr T Kato and Dr M Sasao for fruitful discussions. This work was supported by the Japanese Society for Promotion of Science, Grant No L00537.

\section{Appendix}

Below we list the various mechanisms for population and depopulation of the level with principal quantum number $n$ with the objective to verify the simplification of the CR model employed in the model used. Following [10], the spontaneous radiative decay probability $A_{n_{1}, n_{0}}$ from level $n_{1}$ to level $n_{0}$ can be written as

$$
A_{n_{1}, n_{0}}=A_{0} \frac{g_{0}}{g_{1}} f_{n_{1}, n_{0}}\left(\frac{\Delta E}{R y}\right)^{2}
$$

where $A_{0}=0.80 \times 10^{10} \mathrm{~s}^{-1}, f_{n_{1}, n_{0}}$ is the absorption oscillator strength evaluated using [14], $\Delta E=R y Z^{2}\left(1 / n_{0}^{2}-1 / n_{1}^{2}\right)$ is the transition energy for threshold energy of excitation from $n_{0}$ to $n_{1}, R y$ is the Rydberg constant, and $g_{0}=2 n_{0}^{2}$ and $g_{1}=2 n_{1}^{2}$ are the statistical weights of states with principal quantum numbers $n_{0}$ and $n_{1}$, respectively.

The values $A_{n_{1}, n_{0}}$ of transition from $n_{1}=n_{\mathrm{v}}$ to $n_{0}=n_{\mathrm{v}}-1$ should be much larger than those calculated for excitation and de-excitation rate coefficients for this transition. We used 
the following expression for the excitation rate coefficient (the Van Regemorter formula, see [10]),

$\left\langle v \sigma_{n_{1}, n_{0}}^{\mathrm{exc}}\right\rangle=3.2 \times 10^{-7} f_{n_{1}, n_{0}}\left(\frac{R y}{\Delta E}\right)^{3 / 2} \beta^{1 / 2} \exp (-\beta) G(\beta) \quad\left(\mathrm{cm}^{3} \mathrm{~s}^{-1}\right)$,

and the de-excitation rate coefficient can be obtained from equation (A2) by the detailed balance principle as

$$
\left\langle v \sigma_{n_{1}, n_{0}}^{\mathrm{de}-\mathrm{exc}}\right\rangle=3.2 \times 10^{-7} \frac{g_{0}}{g_{1}}\langle v\rangle f_{n_{1}, n_{0}}\left(\frac{R y}{\Delta E}\right)^{3 / 2} \beta^{1 / 2} G(\beta) \quad\left(\mathrm{cm}^{3} \mathrm{~s}^{-1}\right) .
$$

Here, $\beta$ is defined by $\beta=\Delta E / T_{\mathrm{e}}$, where $T_{\mathrm{e}}$ is the electron temperature. The Gaunt factor $G(\beta)$ is a slowly varying function of $\beta$, with magnitude of the order of unity:

$$
G(\beta)=0.349 f(\beta)+0.0988+0.455 \beta^{3 / 2} f(\beta),
$$

where the function $f$ is taken from [11]

$$
f(\beta)=-\exp (\beta) E i(-\beta) \approx \ln \left(1+\frac{0.562+1.4 \beta}{\beta(1+1.4 \beta)}\right) .
$$

The collisional ionization can be neglected, as its rate coefficient from the level $n$ is much smaller than the radiative decay $A_{n_{1}, n_{0}}$ of this level to the ground state. The radiative recombination rate coefficient to the level $n$ is also much smaller than the $\mathrm{CX}$ recombination rate $N_{\mathrm{H}} N_{\mathrm{H}} \sigma_{n}^{\mathrm{CX}}$. For verifying these statements, one can use the following formulae for the collisional ionization and radiative recombination rate coefficients for the level $n$ [10]:

$$
\begin{aligned}
& \left\langle v \sigma_{n}^{\text {ion }}\right\rangle=6.0 \times 10^{-8} q\left(\frac{R y}{I_{Z}}\right)^{3 / 2} \beta_{Z}^{1 / 2} \exp \left(-\beta_{Z}\right) f\left(\beta_{Z}\right) \quad\left(\mathrm{cm}^{3} \mathrm{~s}^{-1}\right), \\
& \left\langle v \sigma_{n}^{\text {rr }}\right\rangle=5.1 \times 10^{-14} Z \beta_{Z}^{3 / 2} f\left(\beta_{Z}\right) \quad\left(\mathrm{cm}^{3} \mathrm{~s}^{-1}\right),
\end{aligned}
$$

where $q$ is the number of equivalent electrons at the level $n_{\mathrm{v}}$ (for hydrogen-like ions $q=1$ ), $\beta_{Z}=I_{Z} / T_{\mathrm{e}}$ and $I_{Z}=R y(Z / n)^{2}$ is the corresponding binding energy.

Let us compare the rate coefficients (their ratios) of the four processes listed above. In figure $\mathrm{A} 1$, the ratios of the processes which populate and depopulate the level $n_{\mathrm{v}}$ (for the visible domain) of H-like ions of the injected impurity with nuclear charge $Z$ are shown for typical plasma parameters $T_{\mathrm{e}}=2 \mathrm{keV}$ and $N_{\mathrm{e}}=3 \times 10^{13} \mathrm{~cm}^{-3}$. It is seen that all ratios are rather small, which allows us to neglect the corresponding processes in the simplified form of a CR set of equations. It is seen from figure A1 that the ratios are proportional to the electron density $N_{\mathrm{e}}$ and, thus, an unfavourable situation is realized at high densities. From equations (A2), (A3), (A6) and (A7), one can conclude that the rate coefficient values for these atomic processes increase when $T_{\mathrm{e}}$ decreases. Because $A_{n_{1}, n_{0}}$ does not depend on $T_{\mathrm{e}}$, an unfavourable situation is realized at low temperatures. Therefore, we used a plasma electron temperature $T_{\mathrm{e}}=1 \mathrm{keV}$ and electron density $N_{\mathrm{e}}=10 \times 10^{13} \mathrm{~cm}^{-3}$ values to calculate the same ratios of rate coefficients shown in figure A1. These results are shown in figure A2. It is seen from figure A2 that at these plasma parameters the ionization rate from the level $n_{\mathrm{v}}$ by electron collisions becomes comparable with the spontaneous radiative decay probability for low $Z \leqslant 10$ tracer material. It slightly restricts the applicability of the developed model at the low- $Z$, high-density and low electron temperature margin of obtained operational limits shown in figure 9.

In the calculation of simplified ionization balance, one should use the effective (total) rate coefficients of ionization of $\mathrm{H}$ - and $\mathrm{He}$-like ions, radiative recombination of the nuclei and $\mathrm{H}$-like ions, and dielectronic recombination of $\mathrm{H}$-like ions. 


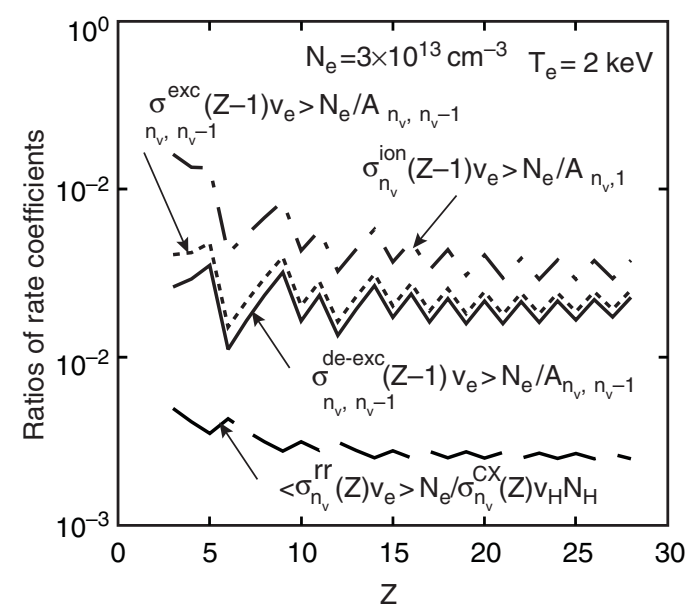

Figure A1. Ratios of rate coefficients for different population and depopulation processes of level $n_{\mathrm{v}}$ versus nuclear charge $Z$ of injected impurity for typical plasma parameters in LHD $\left(T_{\mathrm{e}}=2 \mathrm{keV}\right.$ and $N_{\mathrm{e}}=3 \times 10^{13} \mathrm{~cm}^{-3}$ ).

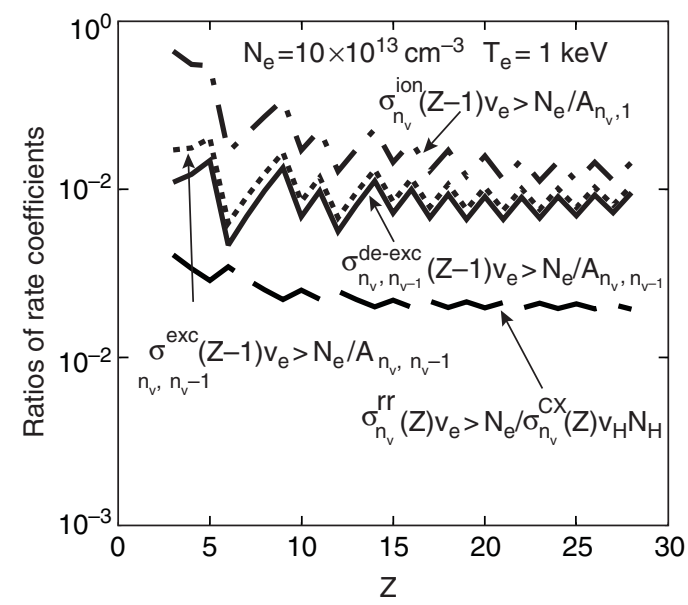

Figure A2. Ratios of rate coefficients for different population and depopulation processes of level $n_{\mathrm{v}}$ versus nuclear charge $Z$ of injected impurity for $T_{\mathrm{e}}=1 \mathrm{keV}$ and $N_{\mathrm{e}}=10^{14} \mathrm{~cm}^{-3}$.

Results of calculations of effective ionization rate coefficients of $\mathrm{H}$-like ions performed by the CR model [10] confirm that they are rather close to those calculated by equation (A6) with $n_{\mathrm{v}}=1$.

For the total rate coefficient of radiative recombination of impurity nuclei with charge $Z$, the formula from [16] was used:

$$
\begin{aligned}
& \left\langle v \sigma_{\mathrm{tot}}^{\mathrm{rr}}(Z)\right\rangle=5.18 \times 10^{-14} Z \xi_{Z} f_{1}\left(\xi_{Z}\right) \quad\left(\mathrm{cm}^{3} \mathrm{~s}^{-1}\right) \\
& \xi_{Z}=\frac{Z}{137} \times \frac{500}{T_{\mathrm{e}}^{1 / 2}(\mathrm{eV})}, \\
& f_{1}\left(\xi_{Z}\right)=\ln \left(\xi_{Z}\right)+0.45+\frac{0.463}{\xi_{Z}^{2 / 3}}+\frac{0.067}{\xi_{Z}^{4 / 3}}+\frac{0.078-0.046 \cdot \ln \left(\xi_{Z}\right)}{\xi_{Z}^{2}}
\end{aligned}
$$


To calculate the total rate coefficient of radiative recombination of $\mathrm{H}$-like ions, we used equation (A8) with charge $Z-1$. An accuracy of about $50 \%$ was found for this approach by comparing the results of accurate calculations of these rate coefficients [15] with the $\left\langle v \sigma_{\text {tot }}^{\mathrm{rr}}(Z-1)\right\rangle$ values obtained from equation (A8).

The rate coefficient for dielectronic recombination from $\mathrm{H}$-like ions to He-like ions was taken from the [17]:

$$
\begin{aligned}
& \left\langle v \sigma^{\mathrm{dr}}(Z)\right\rangle=2.7 \times 10^{-9} \frac{A_{1}(Z)}{\left(T_{\mathrm{e}}(\mathrm{eV})\right)^{3 / 2}} \exp \left(-\frac{A_{2}(Z)}{T_{\mathrm{e}}(\mathrm{eV})}\right) \exp \left(-A_{3}(Z)\right) \quad\left(\mathrm{cm}^{3} \mathrm{~s}^{-1}\right), \\
& A_{1}(Z)=\frac{1230}{Z^{0.14}} \exp \left(-\frac{44}{Z+2.86}\right), \quad A_{2}(Z)=7.5 Z^{2}, \quad A_{3}(Z)=0.0222 Z .
\end{aligned}
$$

\section{References}

[1] Sudo S 1993 Plasma Fusion Res. 691349

[2] Khlopenkov K and Sudo S 2001 Plasma Phys. Control. Fusion 431547

[3] Sudo S et al 2002 Plasma Phys. Control. Fusion 44129

[4] Sergeev V et al 2001 National Institute for Fusion Science Research Report NIFS-710 Japan

[5] Motojima O et al 1999 Phys. Plasmas 61843

[6] Fujiwara M et al 1999 Plasma Phys. Control. Fusion 43 B 157

[7] Yamada H et al 2001 Proc. 13th Eur. Conf. on Control. Fusion and Plasma Phys. (invited paper, see http://www.cfn.ist.utl.pt/EPS2001/)

[8] Ida K, Kado S and Liang Y 2000 Rev. Sci. Instrum. 712360

[9] Janev R K and Smith J J 1993 Suppl. J. Nucl. Fusion 4 No 1

[10] Sobelman I I, Vainshtein L A and Yukov E A 1981 Excitation of Atoms and Broadening of Spectral Lines (Berlin: Springer)

[11] Janev R K, Presnyakov L P and Shevelko V P 1983 Physics of Highly Charged Ions (Berlin: Springer)

[12] Bethe H A and Salpeter E E 1957 Quantum Mechanics of One-and Two-Electron Atoms (Berlin: Springer) p 262

[13] Hulse R A 1982 Fusion Technol. 3259

[14] Johnson L C 1972 Astrophys. J. 174227

[15] Kato T and Asano E 1999 National Institute for Fusion Science Research Report NIFS-DATA-54 Japan

[16] Katkov V M and Strakhovenko V M 1978 Sov. Phys. JETP 48639

[17] Hahn Y 1993 J. Quant. Spectrosc. Radiat. Transfer 4981 